\title{
Experiments on turbulence and near bank vorticities in an open channel sharp bend
}

\author{
Alireza Farhadi $^{1,{ }^{*}}$, Christine Sindelar ${ }^{1}$, Michael Tritthart $^{1}$, and Helmut Habersack ${ }^{1}$ \\ ${ }^{1}$ Institute of Water Management, Hydrology and Hydraulic Engineering, Department of Water, \\ Atmosphere and Environment, University of Natural Resources and Life Sciences (BOKU), Vienna, \\ Austria
}

\begin{abstract}
Turbulence has a significant influence on fluvial processes. Especially in the sharp bends of geometrically complex natural streams, the interaction of turbulence and flow three-dimensionalities is considerable. Here, generation and evolution of streamwise vorticity in an open channel bend is assessed by means of term-by-term experimental investigations. As a result, corresponding mechanisms are shown and analysed. In addition, based on the homogeneity term of vorticity equation, the applicability of a widely used simplified equation of streamwise vorticity is discussed. The presented comparison reaffirms the inadequacy of the equation in order to estimate near bank vorticities.
\end{abstract}

\section{Introduction}

Cross-stream circulations, also known as secondary flows, characterize open-channel flow in bends. In addition to the dominant classic circulation (hereafter main-cell), a weaker counter-rotating cell of circulation is frequently reported in the outer-bank of curved channels $[1,2,3]$. The outer-bank cells (OBC) are of utter importance in bank erosion research. Their role in bank erosions is already discussed [4, 5]; although due to the scarcity of detailed experimental investigations, this issue is still an open discussion.

The main-cell of secondary flow is defined based on the local centrifugal force and the cross-stream pressure gradient in cross-stream momentum equation as explained in [5], which gives the following simplified downstream vorticity equation.

$$
0=-\frac{1}{1+n / R} \frac{\partial}{\partial z}\left(\frac{v_{S}^{2}}{R}\right)+\frac{\partial^{2} \overline{v_{n}^{\prime} v_{z}^{\prime}}}{\partial z^{2}}+v \nabla^{2} \omega_{S}
$$

where $s, n$, and $z$ are curvilinear coordinate system components along the streamwise, lateral and vertical axes, $v_{i}{ }_{i}$ are fluctuating velocity components, $\overline{v_{i}^{\prime} v_{j}^{\prime}}$ are turbulent stresses $(i, j=s, n, z), R$ radius of curvature, $v$ molecular kinematic viscosity; $\nabla^{2}$ is the Laplace operator and $\omega_{s}$ is the streamwise vorticity, which defines as the curl of the velocity vector. Equation 1 shows the balanced between vorticity generator (i.e. centrifugal force) and dissipator (i.e. turbulent shear stress) components. This equation is widely applied in classical hydraulic and engineering practices. Despite the wide usage and good results out

\footnotetext{
${ }^{*}$ Corresponding author: alireza.farhadi@boku.ac.at
} 
of this equation in case of mild bends, there are important concerns about their applicability to the sharp bends $[5,6,7]$. Here, based on a term-by-term approach, the range of discrepancies between the simplified equation (i.e. equation 1) and full equation terms, concerning OBCs zone, is investigated and discussed.

\section{Experimental setup and results}

In order to investigate secondary flows in a sharp bend a styrofoam flume consisting of two straight sections of 2 meters in length and two half circle bends of $180^{\circ}$ with a centreline radius of 1.25 meter (Figure 1.a) was constructed in the Hydraulic Engineering laboratory of the University of Natural Resources and Life Sciences, Vienna. In the flume, flow was induced using a belt drive (Figure 1.b). An Acoustic Doppler Velocimeter (ADV) was used to measure the three-dimensional flow field and corresponding turbulence characteristics. Measurements were conducted over 18 cross-sections, comprising 1024 data points and for three different low Froude numbers (i.e. 0.05-0.16). The size of the measurement grid per cross-section was $33 \mathrm{~mm}$ horizontally and $50 \mathrm{~mm}$ vertically (except for two bottom rows which were distanced by $20 \mathrm{~mm}$ ); the lateral distance of the grid to the banks was $50 \mathrm{~mm}$, the vertical distance to the bottom and to the water surface was $20 \mathrm{~mm}$ and $61 \mathrm{~mm}$, respectively. The flume was characterized by trapezoidal cross-sections (Figure 1.c) within the investigation area (C1-90 to C2-90); in the region of the belt-drive, a rectangular crosssection was present which then expanded continuously into the trapezoidal cross-sections (C1-0 to C1-55 and C2-125 to C2-160); the inner banks of trapezoidal cross-sections were inclined while the outer bank was vertical. Measurement details and data quality controls are provided in $[3,8]$. Hydraulic properties of the flume are presented in Table 1. As an example of sharp open channel bends, where secondary flows are expected to be more pronounced, three cross-sections in the second bend of the racetrack flume (i.e. C2-20, C255 and C2-90) are selected for further investigations.

(a)

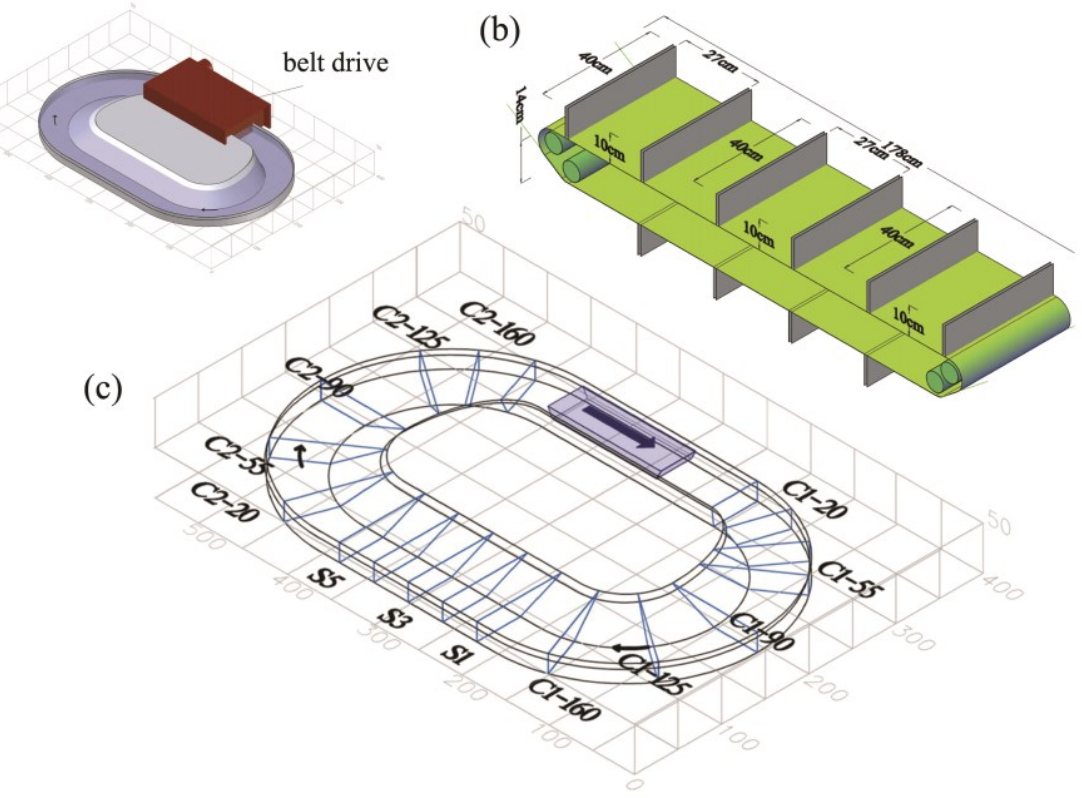

Fig. 1. Racetrack flume (a) an isometric view of the model, (b) a belt drive is used to induce flow, (c) measurement cross-sections and corresponding labels. 
Table 1. Hydraulic properties for triple runs.

\begin{tabular}{ccccccc}
\multirow{2}{*}{ Run } & $Q$ & $\boldsymbol{U}$ & Re & Fr & $u_{*}$ \\
\cline { 2 - 3 } & $\left(1 \mathrm{~s}^{-1}\right)$ & $\left(\mathrm{m} \mathrm{s}^{-1}\right)$ & & & $\left(\mathrm{m} \mathrm{s}^{-1}\right)$ \\
\hline $\mathrm{F}-5$ & 9.9 & 0.067 & $7.6 \times 10^{3}$ & 0.05 & 0.009 \\
$\mathrm{~F}-8$ & 14.5 & 0.098 & $11.1 \times 10^{3}$ & 0.08 & 0.014 \\
$\mathrm{~F}-16$ & 29.1 & 0.197 & $22.3 \times 10^{3}$ & 0.16 & 0.032 \\
\hline
\end{tabular}

$\boldsymbol{U}$ is flume mean velocity, $U=Q /$ Cross-section Area, where $Q$ is flume discharge, $\operatorname{Re}, \mathrm{Fr}$ and $u_{*}$ are Reynolds number, Froude number, and shear velocity respectively, $\mathrm{H}($ depth$)=0.20 \mathrm{~m}, \mathrm{~B}$ (flume-bed width) $=1.00 \mathrm{~m}$.

Vorticity profiles (Figure 2) depict secondary flow patterns for the investigated crosssections. As expected, in addition to the main-cells of secondary flow, the counter-rotating cells are discernible in outer-banks of the cross-sections. The OBCs formation/initiation can be observe from the bend entrance in C2-20 to a developed size and perceptible magnitude in C2-90 (i.e. apex of the bend).
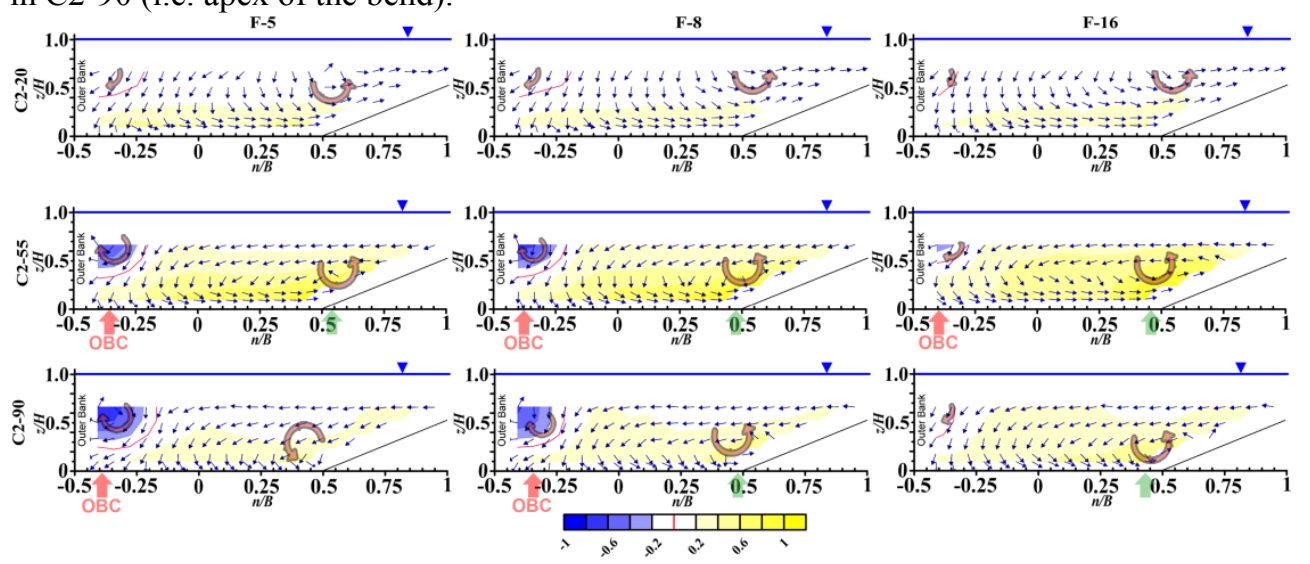

Fig. 2. Vectors of the normalized cross-stream velocity components and (underset) corresponding normalized vorticity $\omega_{s} \mathrm{H} / \mathrm{U}$ profiles. The centre of cross-stream rotation, either OBCs (red) or maincells (green), is marked with upward arrows.

Previously, an extensive investigation [3] was performed concerning mean flow, turbulent kinetic energy exchange and terms of the vorticity balance equation. Based on the mentioned study, OBCs generation is a mixture of "centrifugal force induced vorticities" (Prandtl's first kind of secondary flows, see [9]) and "turbulence induced vorticities" (Prandtl's second kind) mechanisms. The full term downstream vorticity balance equation for a cross-section is provided in [5]. Equation (2) shows the equation neglecting dissipation due to the molecular viscosity term. Also, terms related to the downstream advective transport and the downstream non-uniformity $(\partial / \partial s=0)$ are considered to be not essential [3].

$$
\begin{gathered}
\frac{\partial \omega_{s}}{\partial t}=\underbrace{-\frac{1}{1+n / R} \frac{\partial}{\partial z}\left(\frac{v_{s}^{2}}{R}\right)}_{\text {centrifugal term }}+\underbrace{\frac{\partial^{2}}{\partial z \partial n}\left(\overline{v_{n}^{\prime 2}}-\overline{v_{z}^{\prime 2}}\right)+\frac{1}{1+n / R} \frac{1}{R} \frac{\partial \overline{v_{n}^{\prime 2}}}{\partial z}}_{\text {Anisotropy term }} \\
+\underbrace{\left\{\frac{1}{1+n / R} \frac{\partial^{2}}{\partial z^{2}}-\frac{\partial}{\partial n}\left(\frac{1}{1+n / R} \frac{\partial}{\partial n}\right)\right\}\left[(1+n / R) \overline{v_{n}^{\prime} v_{z}^{\prime}}\right]}_{\text {Homogeneity term }}
\end{gathered}
$$


As for the main-cell of secondary flows, it is expected and already verified $[5,9]$ that the centrifugal term (CFG) functions as vortex generator term and the latter, i.e. anisotropy (ANS) and homogeneity (HOM) terms, is the vortex dissipator term. However, the OBCs generation procedure is following a different pattern. As the mentioned terms in equation 2 are shown in Figure 3, one can visually discern that mentioned explanations for vortex generation and dissipation are inaccurate in the case of outer-bank zones vorticities. Table 2 summarizes the visual perception of Figure 3; it confirms vortex generation by the centrifugal term for all cases and also the degenerative nature of the anisotropy (ANS) term. The anisotropy term in all cases is a degenerative term; however, in the case of F-5 and in comparison to centrifugal term, it shows an order of magnitude lower value. As for the homogeneity (HOM) term, obviously, this term is either a very week degenerative parameter (see results for F-8) or shows positive, i.e. vortex generative, values. Based on the current investigations, the homogeneity term in outer-bank zones of a sharp openchannel shows similar vortex generative behaviour as in straight open channels (Prandtl's second kind of secondary flows) [10].
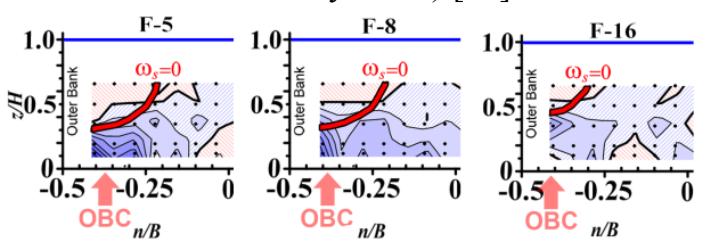

$$
C F G=-\frac{1}{1+n / R} \frac{\partial}{\partial z}\left(\frac{v_{s}^{2}}{R}\right)
$$
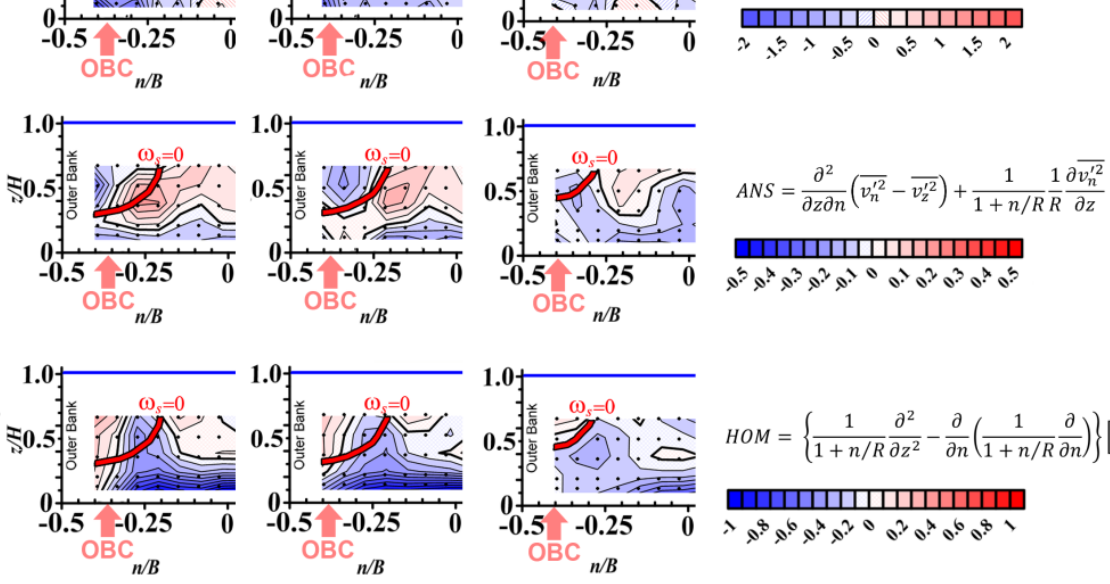

$$
H O M=\left\{\frac{1}{1+n / R} \frac{\partial^{2}}{\partial z^{2}}-\frac{\partial}{\partial n}\left(\frac{1}{1+n / R} \frac{\partial}{\partial n}\right)\right\}\left[(1+n / R) \overline{v_{n}^{\prime} v_{z}^{\prime}}\right]
$$

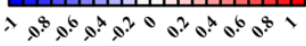

Fig. 3. Terms of downstream vorticity balance equation for the outer-bank zone $(-0.5<\mathrm{n} / \mathrm{B}<0.5)$ of C2-55 (mid-bend) cross-section.

Considering downstream vorticity balance equation and despite the fact that the flow is steady, the sum of CFG, ANS and HOM terms is not zero. These discrepancies could emerge from neglecting the advective transport and non-uniformity $\partial / \partial$ s-terms. Figure 2 shows that the flow is non-axisymmetric; therefore neglecting the downstream nonuniformity term causes misestimation.

Table 2. Mean values (averaged over $\mathrm{OBC}$ zone areas in Figure 3) of downstream vorticity balance equation terms.

\begin{tabular}{c|ccc} 
Term & F-5 & F-8 & F-16 \\
\hline CFG & 0.0445 & 0.0363 & 0.0766 \\
ANS & -0.0088 & -0.0835 & -0.0539 \\
HOM & 0.0132 & -0.0011 & 0.0155 \\
\hline
\end{tabular}




\section{Discussion and Conclusion}

We already presented the simplified downstream vorticity equation (equation 1), which is widely applied in linear models [7]. Linear models do not consider nonlinear feedback between downstream velocity profiles and cells of secondary flow; this often leads to misestimation of vorticty and three-dimensionality in bends [5]. Moreover, it was shown that in sharp bends the homogeneity term (HOM) has an expected vortex generative / degenerative behaviour, which is in contrast to the classical definition of the term for straight open channels.

In fact, the Reynolds shear stress term $\left(\partial^{2} \overline{\mathrm{v}_{\mathrm{n}}^{\prime} \mathrm{v}_{\mathrm{z}}^{\prime}} / \partial \mathrm{z}^{2}\right)$, i.e. turbulence term in equation 1 , is the reduced form of the homogeneity term in equation 2 . Therefore, it would be interesting to see the extent of data which will be lost by applying simplified equation of vorticity. The corresponding normalized profiles of the homogeneity term (HOM) and the turbulence term of the simplified streamwise vorticity equation are provided in Figures (4-6). Notwithstanding the fact that the general mid-channel zone's patterns of HOM and the turbulence term are similar, it is notable that as the flow proceeds through the bend from (C2-20) to (C2-90), the differences between the mentioned terms are increasing. This is particularly evident for outer bank zones (dark shadow squares in the Figures 4-6).
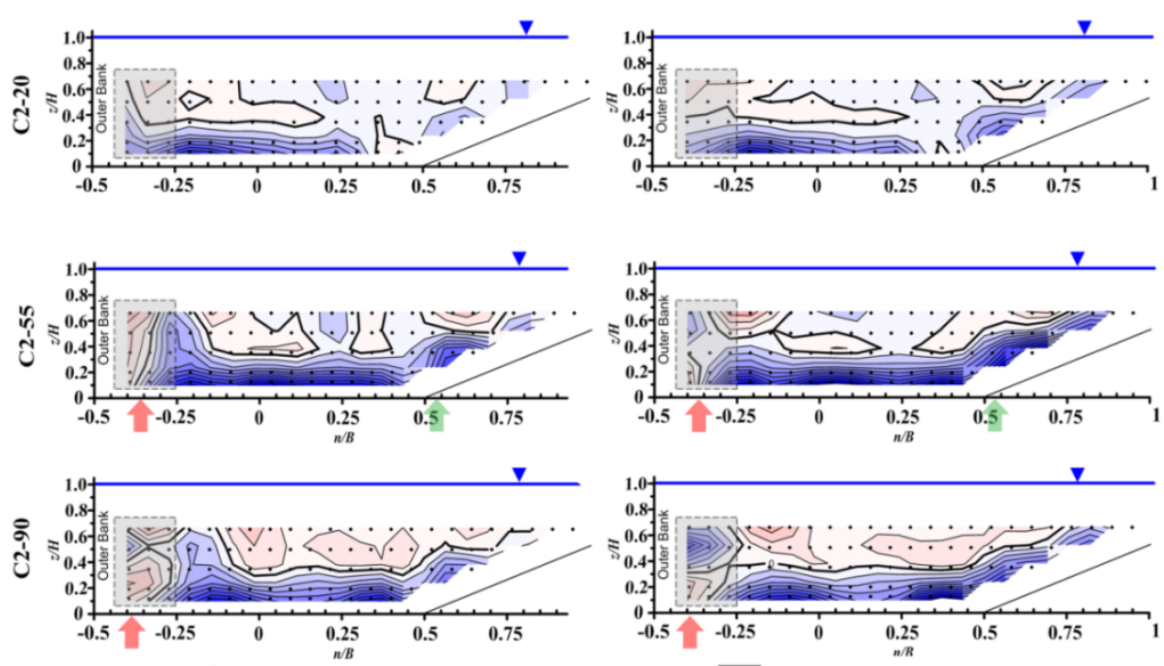

(a) $\left\{\frac{1}{1+n / R} \frac{\partial^{2}}{\partial z^{2}}-\frac{\partial}{\partial n}\left(\frac{1}{1+n / R} \frac{\partial}{\partial n}\right)\right\}\left[(1+n / R) \overline{v_{n}^{\prime} v_{z}^{\prime}}\right]$

(b) $\frac{\partial^{2} \overline{v_{n}^{\prime} v_{z}^{\prime}}}{\partial z^{2}}$

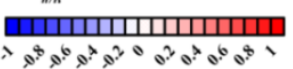

Fig. 4. Normalized profiles of (a) the homogeneity term $/\left(\mathrm{U}^{2} / \mathrm{H}^{2}\right)$ comparing to (b) the turbulent term in simplified streamwise vorticity equation $/\left(\mathrm{U}^{2} / \mathrm{H}^{2}\right)$ for $\mathbf{F}-\mathbf{5}$. The red and green upward arrows indicate the approximate location of the $\mathrm{OBC}$ and main cell centre respectively. Shaded areas show approximate $\mathrm{OBC}$ zones.

The applicability of the simplified equation of streamwise vorticity was already questioned with regard to the main cell of secondary flows for strongly curved channels [5, 7]. The comparison presented in this work, therefore, reaffirms the inadequacy of this equation in order to estimate outer bank zone vorticities. As a result, turbulent induced vorticities in sharp open channels cannot be resolved using linear models. In the case, one should decide whether the near-bank vortices prediction is crucial for a certain project, concerning the approach and scale of the work. As for large-scale river investigations, one can neglect such phenomena; however, as mentioned before such processes are crucial and most are not addressed properly in hydromorphological fluvial studies. 

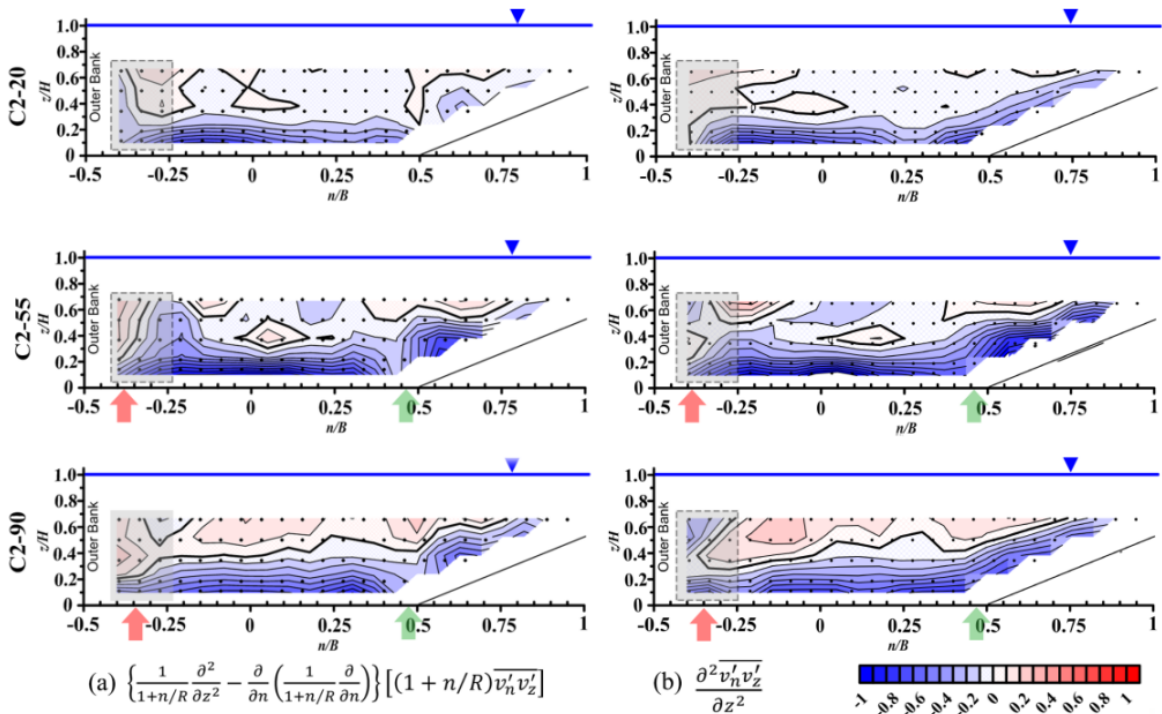

Fig. 5. Normalized profiles of (a) the homogeneity term $/\left(\mathrm{U}^{2} / \mathrm{H}^{2}\right)$ comparing to (b) the turbulent term in simplified streamwise vorticity equation $/\left(\mathrm{U}^{2} / \mathrm{H}^{2}\right)$ for $\mathbf{F - 8}$. The red and green upward arrows indicate the approximate location of the $\mathrm{OBC}$ and main cell centre respectively. Shaded areas show approximate $\mathrm{OBC}$ zones.
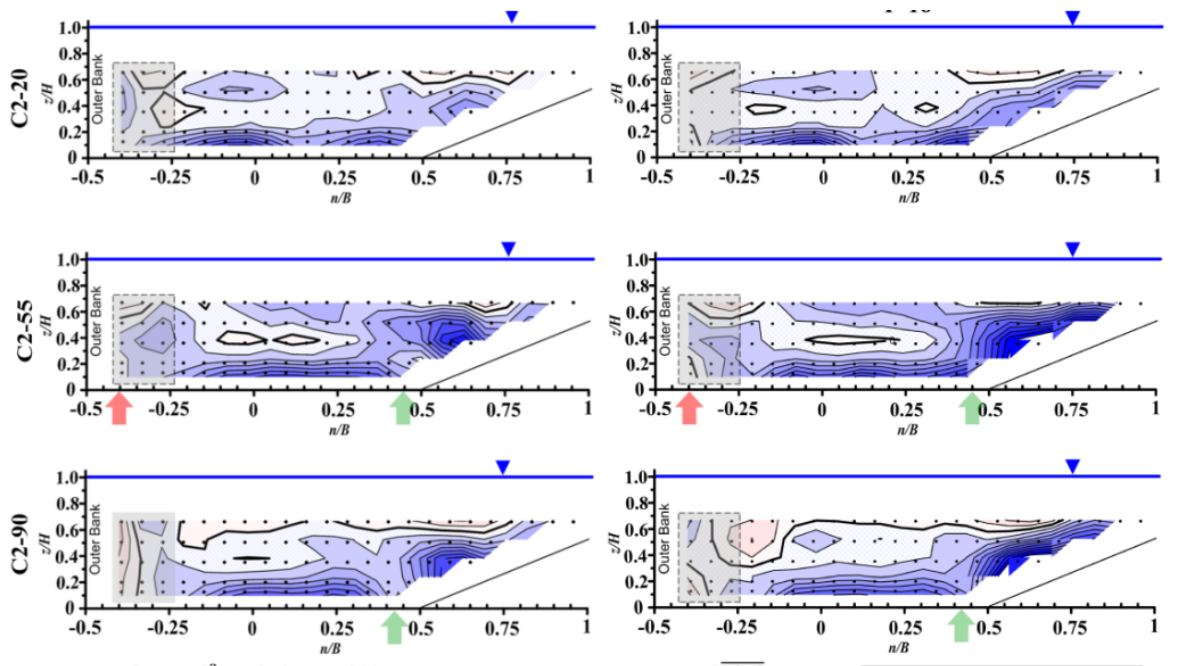

(a) $\left\{\frac{1}{1+n / R} \frac{\partial^{2}}{\partial z^{2}}-\frac{\partial}{\partial n}\left(\frac{1}{1+n / R} \frac{\partial}{\partial n}\right)\right\}\left[(1+n / R) \overline{v_{n}^{\prime} v_{z}^{\prime}}\right]$

(b) $\frac{\partial^{2} \overline{v_{n}^{\prime} v_{z}^{\prime}}}{\partial z^{2}}$

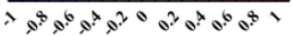

Fig. 6. Normalized profiles of (a) the homogeneity term $/\left(\mathrm{U}^{2} / \mathrm{H}^{2}\right)$ comparing to (b) the turbulent term in simplified streamwise vorticity equation $/\left(\mathrm{U}^{2} / \mathrm{H}^{2}\right)$ for $\mathbf{F}-\mathbf{1 6}$. The red and green upward arrows indicate the approximate location of the $\mathrm{OBC}$ and main cell centre respectively. Shaded areas show approximate $\mathrm{OBC}$ zones.

Additionally, it seems that neglecting the advective transport and the assumption of non-essentiality of downstream non-uniformity term, i.e. $\partial / \partial \mathrm{s}=0$, as applied to the full term vorticity balance equation [3,5], is not valid for the experimental results presented in this 
paper. This is in line with Large Eddy Simulations (LES) of a sharp bend in [11]. Therefore, considering the extreme three-dimensionality of the vorticity in bends [12], a detailed three-dimensional volumetric measurement in open channel sharp bends is necessary. Such measurement can provide streamwise and cross-stream parameters in a comparable and applicable format.

\section{References}

1. K. Blanckaert, W. H. Graf, J. Hydraul Eng., 127(10), 835-847 (2001).

2. S. Kang, F. Sotiropoulos, J. Geophys. Res.-Earth, 116(F3) (2011).

3. A. Farhadi, C. Sindelar, M. Tritthart, M. Glas, K. Blanckaert, H. Habersack, J. HydroEnviron. Res., 18, 1-11 (2018).

4. B. Christensen, K. Gislason, J. Fredsoe, 1st RCEM Symp., 543-553 (1999).

5. K. Blanckaert, H. J. De Vriend, J. Fluid Mech., 498, 353-380 (2004).

6. R. Booij, J. Tukker, Developments in Laser Techniques and Applications to Fluid Mechanics, 98-114 (Springer, Berlin, 1996).

7. A. Farhadi, A. Mayrhofer, M. Tritthart, M. Glas, H. Habersack, Eng. Appl. Comp. Fluid, 12(1), 216-235 (2018).

8. M. Glas, M. Tritthart, B. Zens, H. Keckeis, A. Lechner, T. Kaminskas, H. Habersack, Can. J. Fish Aquat. Sci.IS, 74(9), 1474-1489 (2017).

9. I. Nezu, H. Nakagawa, Turbulence in open channels (Balkema, Rotterdam, The Netherlands, 1993).

10. P. Bradshaw, Annu. Rev. Fluid Mech., 19(1), 53-74 (1987).

11. W. Van Balen, K. Blanckaert, W. S. Uijttewaal, J. Turb., 11, N12 (2010).

12. M. Sanjou, I. Nezu, J. Hydraul. Res., 47(5), 598-610 (2009). 